\title{
A molecular dynamics simulation study for the mechanical properties of different types of carbon nanotubes
}

\author{
Keka Talukdar • Apurba Krishna Mitra
}

Received: 30 October 2011/Accepted: 30 March 2012/Published online: 5 May 2012

(c) The Author(s) 2012. This article is published with open access at Springerlink.com

\begin{abstract}
Carbon nanotubes have caught tremendous attention of the researchers during the last decade due to their excellent mechanical, electrical, optical and thermal properties. The exploitation of these fibers as reinforcing agents in making strong fiber composites has been a primary research topic in the recent investigations on composite materials. Although the theoretical results are rather optimistic, the goal of achieving high strength of the carbon nanotube composites is still not satisfactorily realized. We report here a comparative study of the mechanical properties of single-walled, multi-walled and bundle of single-walled carbon nanotubes. Their mechanical behavior is investigated by molecular dynamics simulation, considering Brenner's second generation reactive empirical bond order interatomic potential between the carbon atoms making a tube. For a long range interaction, we have defined a weak van der Waals force which acts between different layers of a multi-walled tube or between different tubes of a bundle. Samples of three isolated armchair single-wall carbon nanotubes of different diameters, a multi-wall armchair carbon nanotube and finally a bundle of three armchair single-walled nanotubes of same diameter are taken. Their fracture pattern and buckling behavior are modeled and compared. Significant changes are observed in the mechanical properties of the samples of different types of carbon nanotubes which arise due to the interaction between the shells of a multi-walled tube or the tubes in a bundle.
\end{abstract}

\footnotetext{
K. Talukdar · A. K. Mitra ( $₫)$

Department of Physics, NIT Durgapur,

Durgapur 713209, West Bengal, India

e-mail: akmrecdgp@yahoo.com

K. Talukdar

e-mail: keka.talukdar@yahoo.co.in
}

Keywords SWCNT - SWCNT bundle $\cdot$ MWCNT . Mechanical properties · MD simulation · Fracture

\section{Introduction}

In the ongoing research on the composite science and technology, carbon nanotubes (CNTs) have earned special attention due to their high strength, high ductility, light weight and high electrical and thermal conductivity. When the mechanical properties are concerned, they can be potentially used as reinforcing agents for making strong and useful composites in automobile industry or in space elevators etc. Mechanical properties of CNTs have been investigated by many researchers both experimentally (Treacy et al. 1996; Wong et al. 1997; Krishnan et al. 1998; Demczyk 2002) and theoretically (Yakobson et al. 1996; Liew et al. 2004; Batra and Sears 2007; Coluci et al. 2007; Avila and Lacerda 2008; Mashapa and Ray 2010; Mohammadpour et al. 2011; Tang et al. 2011). Large variation of calculated values is observed for the mechanical characteristics. Experimental data (Yu et al. 2000) for single-walled carbon nanotube (SWCNT) bundles under tensile loading show that the Young's modulus can be as high as $1.47 \mathrm{TPa}$ and tensile strength may range from 13 to $52 \mathrm{GPa}$. Also, the maximum breaking strain can be $5.3 \%$. Falvo et al. (1997) showed experimentally how a SWCNT bundle bent or buckled under large strains. A transition of the cross section of the tubes in a SWCNT bundle as well as the change of the bulk elastic properties is reported by Liu et al. (2005). The experiments established that the measured lower values of Young's modulus and tensile strength of CNTs were mainly due to the presence of imperfections in them. Mechanical properties of multi-walled carbon nanotubes (MWCNTs) were 
also studied (Nichols et al. 2007; Xia et al. 2007; Zou et al. 2009).

In spite of so many attempts to explain the discrepancies between the theoretical and experimental data regarding the mechanical properties of SWCNTs, some observed discrepancies between theory and experiment remain still unanswered. SWCNTs have a natural tendency to form bundles and thus in the most of the experiments undertaken by various investigators, SWCNT bundles or MWCNTs have been used as samples. So, we must take into account the influence of bundle formation or the interaction in between the shells of an MWCNT in our calculations of mechanical properties of the CNTs, such that we can compare our result well with the experimental observations. We can also have an idea of the influence of interlayer interaction on their mechanical characteristics comparing the properties of SWCNTs, MWCNTs and SWCNT bundles.

\section{Methods and modeling}

Second generation reactive empirical bond order potential (REBO) is adopted to describe the carbon-carbon bonding in the nanotubes.

In the 2nd generation REBO potential (Brenner et al. 2002), the form of the potential is

$V\left(r_{i j}\right)=f_{\mathrm{c}}\left(r_{i j}\right)\left[V^{\mathrm{R}}\left(r_{i j}\right)+b_{i j} V^{\mathrm{A}}\left(r_{i j}\right)\right]$

where

$V^{\mathrm{R}}\left(r_{i j}\right)=f_{\mathrm{c}}\left(r_{i j}\right)\left(1+Q_{i j} / r_{i j}\right) A_{i j} e_{i j}^{-\alpha} r_{i j}$

and

$V^{\mathrm{A}}\left(r_{i j}\right)=-f_{\mathrm{c}}\left(r_{i j}\right) \sum B_{n} e^{-\beta_{i j n} r_{i j}}$

where $f_{\mathrm{c}}\left(r_{i j}\right)$ is a cut-off function that reduces to zero interaction beyond $2.0 \AA$.

$V^{\mathrm{R}}\left(r_{i j}\right)$ is a pair-wise term that models the core-core and electron-electron repulsive interactions and $V^{\mathrm{A}}\left(r_{i j}\right)$ is a pair-wise term that models core-electron attractive interactions, where $r_{i j}$ is the distance between nearest neighbor atoms $i$ and $j$, and $b_{i j}$ is a many-body, bond order term that depends on the number and types of neighbors and the bond angles and bicubic spline.

In order to incorporate a long range of non-bonding interaction, Lennard-Jones (1924) potential is taken which is given by,

$V_{\mathrm{LJ}}=4 \varepsilon_{i j}\left[\left(\sigma_{i j} / r_{i j}\right)^{12}-\left(\sigma_{i j} / r_{i j}\right)^{6}\right]$

$\varepsilon$ is the depth of the potential well, $\sigma$ is the distance at which the inter-particle potential is zero and $r$ is the distance between the particles. $i, j$ stand for $i$ th and $j$ th atoms. The term $r^{-12}$ describes Pauli repulsion at short ranges due to overlapping of electronic orbitals and $r^{-6}$ describes the long range attraction. The L-J potential is truncated at the cut-off distance

$r_{\mathrm{c}}=2.5 \sigma$

where

$$
\begin{aligned}
V\left(r_{\mathrm{c}}\right) & =V(2.5 \sigma)=4 \varepsilon_{i j}\left[\left(\sigma_{i j} / 2.5 \sigma_{i j}\right)^{12}-\left(\sigma_{i j} / 2.5 \sigma_{i j}\right)^{6}\right] \\
& =-0.0163 \varepsilon=-1 / 61.3 \varepsilon .
\end{aligned}
$$

Hence, at $r_{\mathrm{c}}=2.5 \sigma$, the potential $V$ is about $1 / 60$ th of its minimum value. Interactions between two different types of atoms use a geometric average of the $\varepsilon$ values of each atom and a straight average $\sigma$. The Lennard-Jones potential is set to zero for $r_{i j}>2.5 \sigma_{i j}$ and in the range $r_{i j}<R_{i j}^{(2)}$ in which the bond order potential is nonzero. In the range $R_{i j}^{(2)}<r_{i j}<0.95 \sigma_{i j}$, the potential is furthermore replaced by a cubic term which vanishes quadratically at $R_{i j}^{(2)}$ and which meets the Lennard-Jones expression with continuous first derivative at $0.95 \sigma_{i j}$. So, simulation is carried out taking the following form of the potential

$V_{\mathrm{MD}}(r)=\left(\begin{array}{ll}V_{\mathrm{LJ}}(r)-V_{\mathrm{LJ}}\left(r_{\mathrm{c}}\right) & \text { for } r \leq r_{\mathrm{c}} \\ 0 & \text { for } r>r_{\mathrm{c}}\end{array}\right)$

Instead of a simple cut-off, this scheme of truncation and shifting of the potential is physically reasonable. Here, the force smoothly comes to zero value; therefore, the discontinuity at $r=r_{\mathrm{c}}$ is eliminated. Otherwise, in simple truncation method, the force is undefined at the cut-off region and energy is not conserved. So in the present method, the problem of energy conservation is overcome.

The open ends of the CNTs are satisfied by dangling bonds rather than by any other atom. Simulation is performed for each tube. Keeping one end fixed other end is stretched gradually by small strain increments. No other constraint is there in any other direction. When stretching the MWCNT or the bundle, all the tubes are stretched equally. To study the buckling properties, compressive force is applied from one end. Stress is calculated from the energy-strain curve as

$\sigma=1 / V(\mathrm{~d} E / \mathrm{d} \varepsilon)$

where $\sigma$ is the longitudinal stress, $V$ the volume of the tube, $\varepsilon$ the strain and $E$ the strain energy of the tube. Volume of a single tube is

$V=2 \pi r \delta r l$

where $r$ is the inner radius of the tube, $\delta r$ its wall thickness and $l$ the length of the tube. We have taken $\delta r$ as $0.34 \mathrm{~nm}$, which has been the standard value, used by most of the 
authors. For a SWCNT bundle and the MWCNT, the volume of each tube is calculated separately and then added to get the total volume. To calculate stress from the energy strain curve, we have used a linear relationship for the elastic region and appropriate non-linear equations for the segments of high strain deformation regions. Young's modulus is found from the slope of the linear portion of the stress-strain curve. Temperature of the system is maintained at $300 \mathrm{~K}$ by Berendsen thermostat (1984).

\section{Results and discussion}

All the armchair SWCNTs show high tensile strength with a maximum of $196.30 \mathrm{GPa}$ for a $(5,5)$ tube. The stressstrain curves for a $(5,5)$ and a $(10,10)$ SWCNT are stiffer than the curve for a $(7,7)$ tube. The $(7,7)$ tube exhibits some ductility before failure as the stress-strain curve contains a plastic flow region. However, all the curves show sharp fall of stress on failure (the curves are derived from the energy-strain curves which also show sharp fall of energy just after the breaking strain). Looking at their sudden fall of energy, we can conclude that the fracture may be treated as brittle. Stress strain curves of the armchair tubes are merged in Fig. 1. Stress-strain curves under compressive loading follow the same pattern as the curves of Fig. 4.

Failure patterns are modeled in Fig. 2. The breaking of a $(10,10)$ tube is accompanied with deformations in different places. The whole structure of the tube is changed (Fig. 2c). The narrowest tube, i.e., the $(5,5)$ SWCNT breaks from the middle (Fig. 2a), whereas for the $(7,7)$ tube, breaking starts near the edge of the tube (Fig. 2b). Buckling of the armchair tubes is associated with

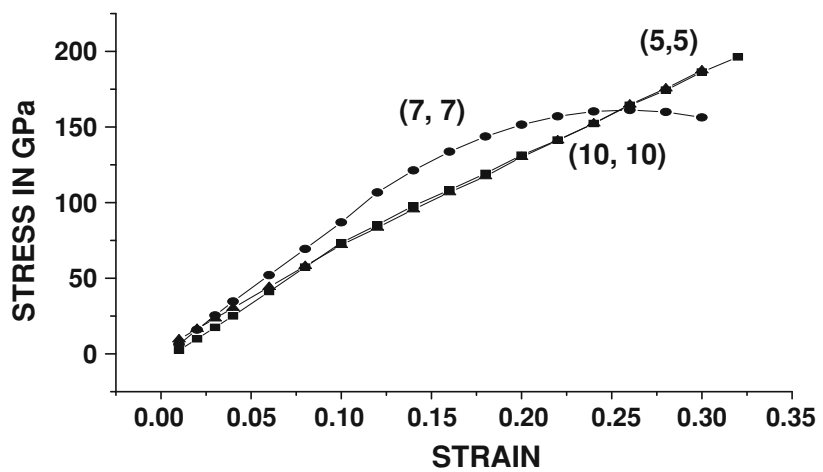

Fig. 1 Stress-strain curves of the armchair SWCNTs under tensile loading

depression and humps in some places. More or less, they follow the same buckling style. The tensile and compressive properties of the SWCNTs are tabulated in Table 1.

When an armchair three-walled CNT is strained and simulated considering long range van der Waals interaction playing between the shells, all the shells are strained in the same manner at first and then they are deformed in different ways after a certain strain. It is observed that the outer wall breaks first and then the inner walls are affected. The whole tube is ruptured when the innermost tube breaks.

The maximum tensile stress is observed as $168.98 \mathrm{GPa}$ with a failure strain of $31 \%$ for this MWCNT (Fig. 3). Figure 4 describes the stress-strain curves for compressive loading. The breaking and buckling of the MWCNT is depicted in Fig 5. It is clear from Fig 5a that outer shells are ruptured completely before the breakage of the innermost wall. Due to the presence of van der Waals attractive force, all the shells of the MWCNT buckle in the same way and the tube is depressed at the middle portion (Fig. 5b).
Fig. 2 Breaking pattern of a $\mathbf{a}(5,5), \mathbf{b}(7,7)$ and $\mathbf{c}(10,10)$ SWCNT. d Buckling of a (10, 10) SWCNT
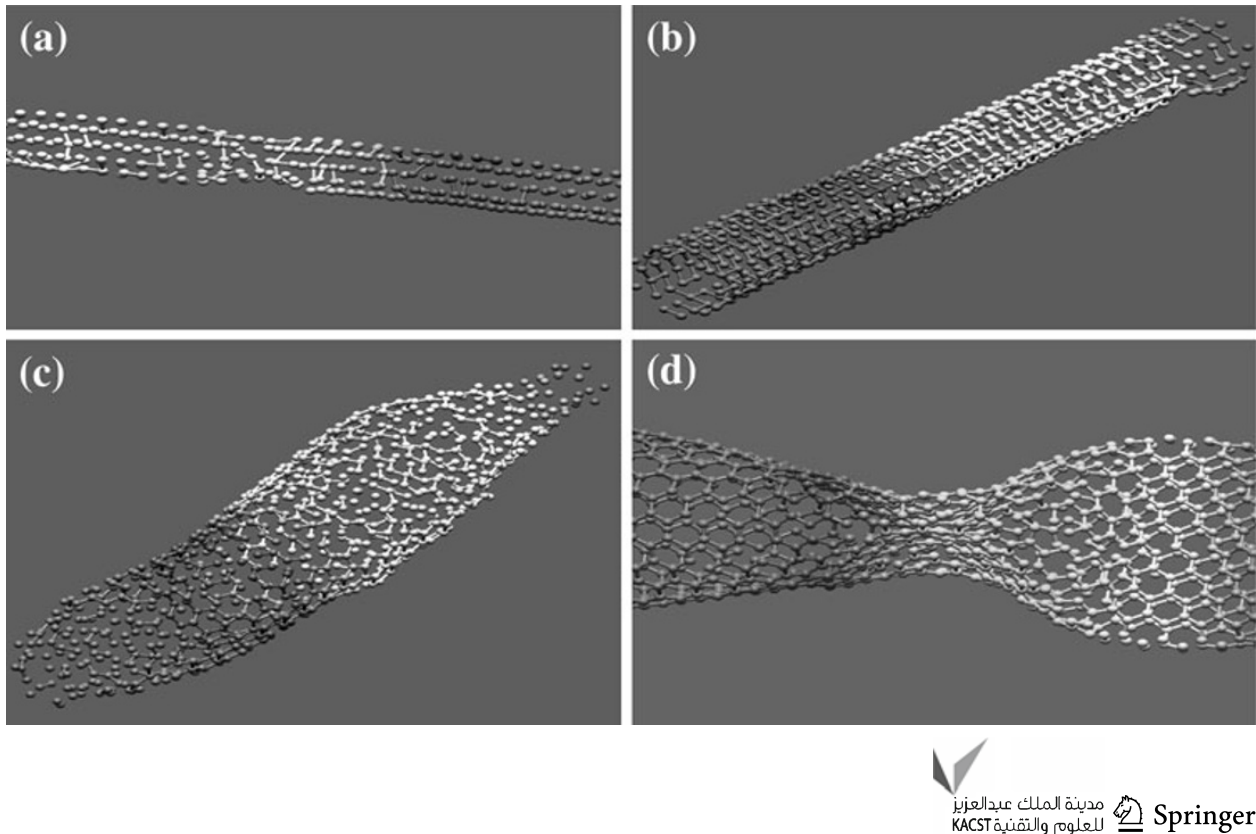
Table 1 Tensile and compressive properties of different types of CNTs

\begin{tabular}{|c|c|c|c|c|c|c|}
\hline \multirow[t]{2}{*}{ CNT type } & \multicolumn{3}{|c|}{ Tensile properties } & \multicolumn{3}{|c|}{ Compressive properties } \\
\hline & Y.M. (Tpa) & M.T.S. (GPa) & F.Sr. $(\%)$ & C.M. (Тpa) & C.B.S (GPa) & C.B.Sr. $(\%)$ \\
\hline$(5,5)$ & 0.79 & 196.91 & 31 & 1.20 & 73.71 & 6 \\
\hline$(7,7)$ & 0.89 & 184.66 & 30 & 1.11 & 69.55 & 7 \\
\hline$(10,10)$ & 0.75 & 161.18 & 24 & 1.13 & 67.02 & 6 \\
\hline Armchair MWCNT & 0.79 & 168.24 & 31 & 0.88 & 98.26 & 11 \\
\hline SWCNT bundle & 0.87 & 112.03 & 21 & 0.99 & 53.66 & 5 \\
\hline
\end{tabular}

Y.M. Young's modulus, M.T.S. maximum tensile strength, F.Sr. failure strain, C.M. compressive modulus, C.B.S. critical buckling strength, C.B. Sr. critical buckling strain

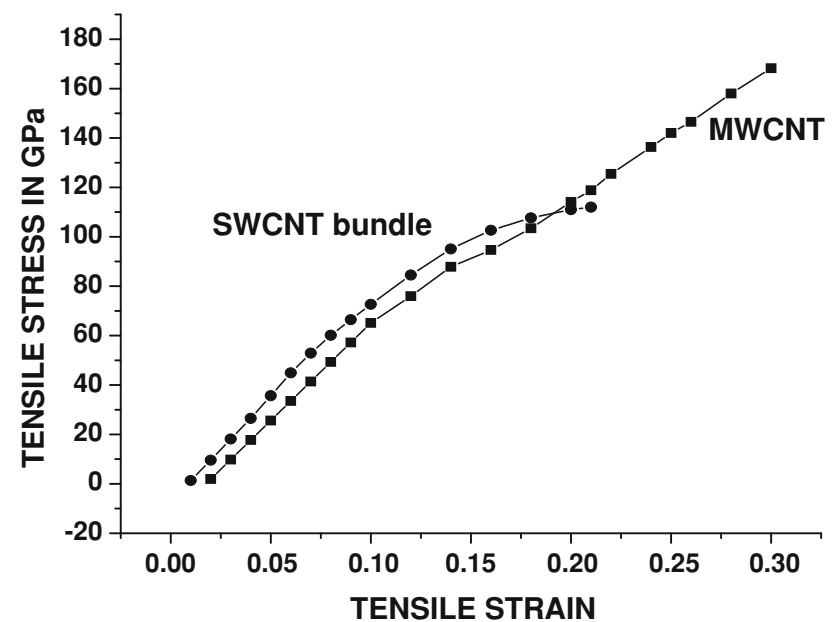

Fig. 3 Stress-strain curves for an armchair MWCNT and a bundle of three armchair SWCNTs under tensile loading

The deformation of the cross section is observed for all the three shells of the MWCNT on the application of pressure. The cross sections are more and more deformed when applied pressure is increased (Fig. 5c). After a certain limit, the atoms in the cross section area are separated from the tube. At this stage, the tube is collapsed completely due to vigorous compression and buckling.

The maximum tensile stress is lower in case of the armchair MWCNTs comprising three $(5,5)$ SWCNTs than the maximum tensile stress of an isolated $(5,5)$ tube. This is a result of van der Waals interaction in between the different shells. Breakage of bond starts much before the complete rupture of the tube due to the influence of other shells in proximity. So, the applied force succeeds over the inner resistive force of the tube against any deformation and the energy of the system comes to a minimum value more easily than that of the energy of an isolated tube. For an isolated tube, the system energy slowly decreases to zero when an external force is applied.

Now the result will be more affected if the three $(5,5)$ SWCNTs form a bundle. The SWCNTs that are grown by laser ablation, arc discharge, or the most recent HiPCO

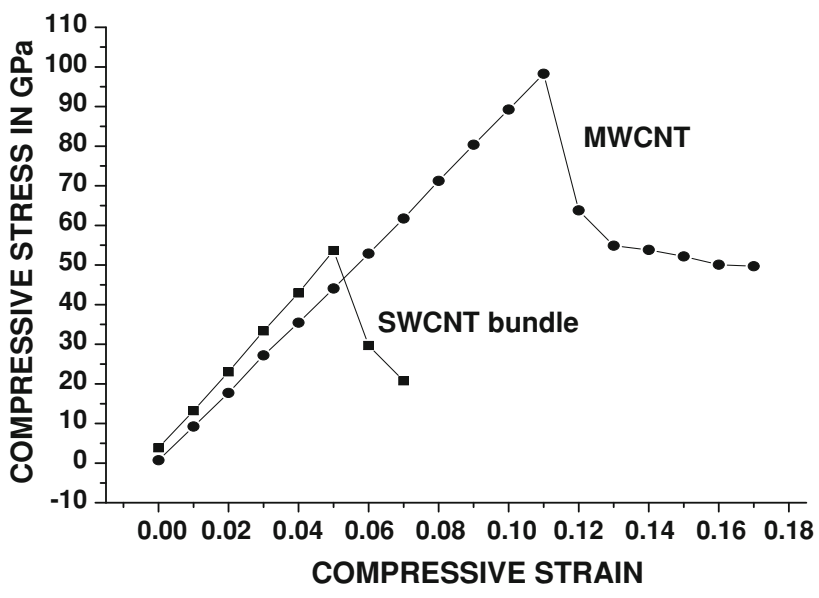

Fig. 4 Stress-strain curves for an armchair MWCNT and a bundle of three armchair SWCNTs under compressive loading

method always occur in bundles. These bundles are held together by weak interactions between the tubes. Bundling also changes their property by tube interaction. A bundle of three armchair $(5,5)$ SWCNTs is shown in Fig. 6. To visualize the whole bundle, the projection of the picture is adjusted and that is why it appears to be of shorter length than actually it is.

The stress-strain behavior of this bundle (Fig. 3) points toward the facts that the mechanical behavior of the CNT changes to a large extent in bundle formation. The failure stress is dropped to $112.03 \mathrm{GPa}$. Also it has the lowest ductility with a maximum tensile strain of $21 \%$, whereas the other tubes have failure strains more than $30 \%$. The same trend of lower critical buckling stress and critical buckling strain is observed for the bundle (Fig. 4). The mechanical properties are all given in Table 1 below. In this calculation, the young's modulus values are in agreement with the experimental results of Yu et al. (2000); Salvetat et al. (1999a, b) and Poncharal et al. (1999). Failure stress values are also in the experimental range obtained by Demczyk et al. (2002). A comparison of the calculated values and 
Fig. 5 a Breaking of the armchair MWCNT, b buckling of the MWCNT (the middle portion is depressed). $\mathbf{c}$ The cross section of the shells of the MWCNT is deformed on the application of compressive force
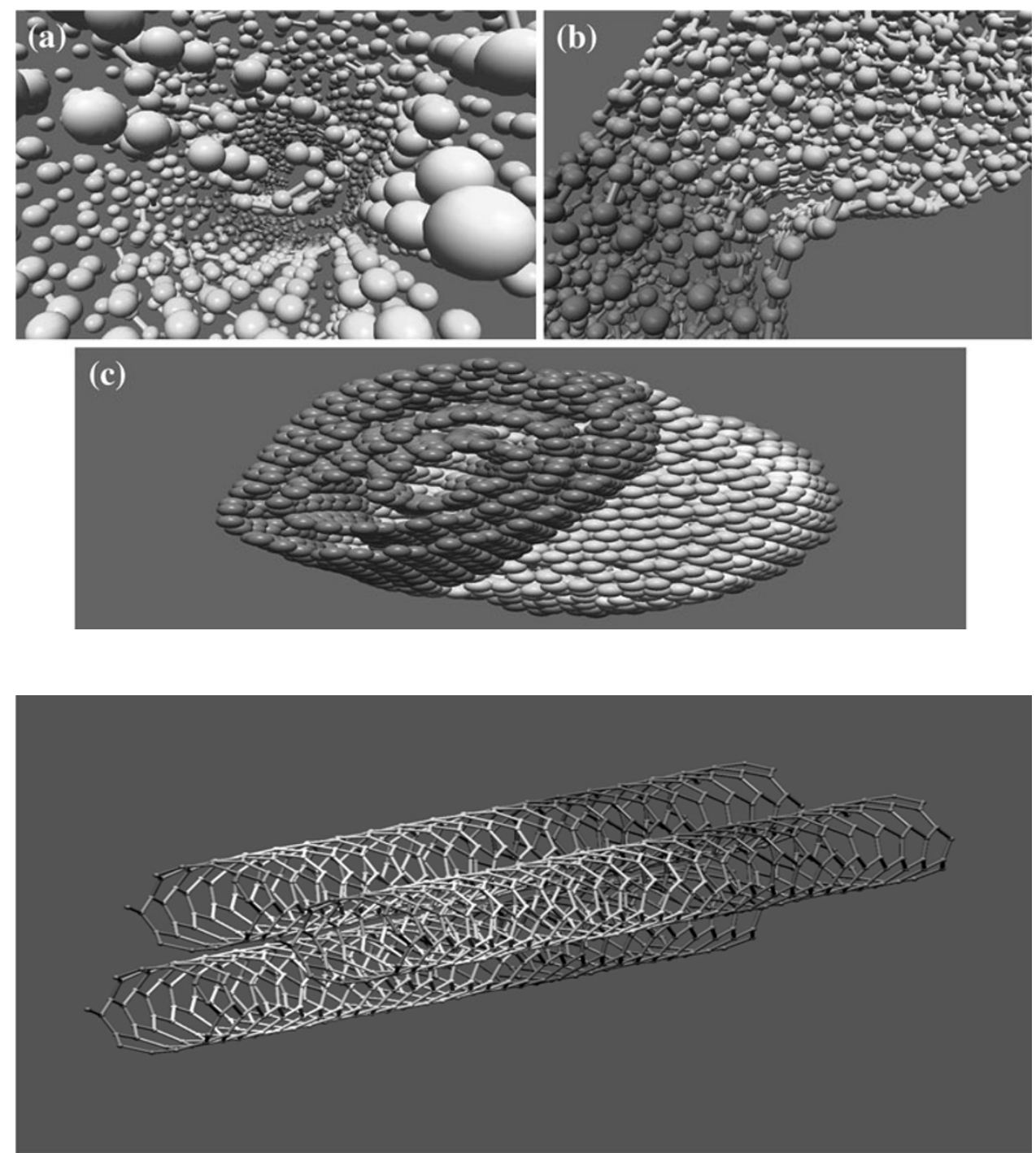

Fig. 6 Picture of an SWCNT bundle composed of three $(5,5)$ SWCNTs

Table 2 Comparison of the calculated values with experimental values of different authors

\begin{tabular}{lllr}
\hline $\begin{array}{l}\text { Y.M. } \\
\text { (calculated value) }\end{array}$ & Y.M. (experimental value) & $\begin{array}{l}\text { M.T.S. } \\
\text { (calculated value) }\end{array}$ & M.T.S. (experimental value) \\
\hline $0.75-0.89 \mathrm{TPa}$ & (1) $0.32-1.47 \mathrm{TPa}$ for SWCNT rope, & $112.03-196.91 \mathrm{GPa}$ & $150 \pm 45 \mathrm{GPa}$ for MWCNTs \\
& $0.27-0.95 \mathrm{TPa}$ for MWCNTs (Yu et al. 2000) & & \\
& (2) $0.81 \pm 0.41$ TPa for MWCNTs & & \\
& (Salvetat et al. 1999a, b) & & \\
& (3) $0.1-1$ TPa for MWCNTs & & \\
& (Poncharal et al. 1999) & & \\
\end{tabular}

experimental values is given in Table 2. For SWCNT bundle, the values of failure stress and strain are dropped down to much lower value which was estimated higher for a single tube.

The breaking of this bundle occurs very symmetrically and starts nearly halfway in between the tube bundle, though the breaking point is slightly closer to the loading edge. All the tubes are equally broken which is shown in
Fig. 7a. Figure $7 \mathrm{~b}$ gives the picture of the buckling pattern of the same.

This changed behavior may be explained by overlapping of energy bands in nanotube bundles. Scanning tunneling spectroscopy (Wildoer et al. 1998), resonant Raman scattering (Jorio et al. 2001) and optical absorption or emission measurements (Connell et al. 2002) confirmed the electronic DOS in carbon nanotubes. Ouyang et al. (2001,

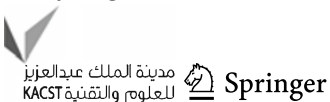


Fig. 7 a Breaking and b buckling of a bundle of three armchair SWCNTs
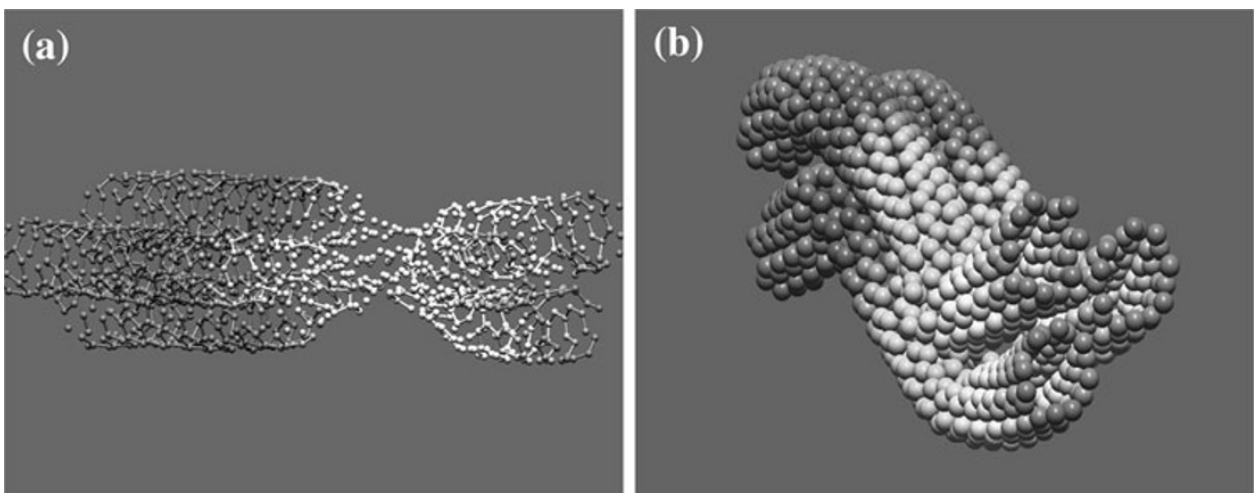

2002) experimentally found a pronounced dip in the electronic DOS of a $(8,8)$ SWCNT at Fermi level inside a bundle, compared to an isolated tube. Due to high symmetry of a metallic armchair SWCNT, the measured DOS was nonzero and constant at Fermi energy. The local DOS measured for an $(8,8)$ SWNT packed in the bundle showed a clear suppression around Fermi level, in contrast to the data for the isolated $(8,8)$ SWNT. This is due to symmetry breaking by other tubes in proximity. Not always a gap is produced, but gap may also be closed for such a tube. Closing of band gap is observed for a $(10,0)$ tube in a bundle by Reich et al. (2002). Due to this type of overlapping of electronic bands, attraction or repulsion arises inside the SWCNT bundle. All intermolecular/van der Waals forces are anisotropic which means that they depend on the relative orientation of the molecules. The induction and dispersion interactions are always attractive, irrespective of orientation, but the electrostatic interaction changes sign upon rotation of the molecules. Due to the overlapping of electronic bands, attraction or repulsion arises inside the SWCNT bundle. Electrostatic interaction changes sign upon rotation of the molecules. That is, the electrostatic force can be attractive or repulsive, depending on the mutual orientation of the molecules giving rise to different types of interaction between the tubes of a bundle. In the low energy part of the band structure, the bundling of the nanotubes changes the electronic properties by symmetry breaking and by the intratube dispersion perpendicular to $K_{\mathrm{z}}$. That also holds good for larger electronic energies also. Again, when isolated tubes of different symmetries are present, energy bands are strongly split. Reich et al. (2002) showed that in a high symmetry packing of $(6,6)$ nanotube, the degenerated bands of isolated tube remain degenerate by symmetry in the crystal. In their study, it was also revealed that the dispersion of the electronic bands perpendicular to $k_{\mathrm{z}}$ is less in a zigzag $(10,0)$ tubes than in a $(6,6)$ tubes. The first two valance states at the $\Gamma$ point of the brillouin zone result in a strong dispersion in the corresponding states perpendicular to $k_{\mathrm{z}}$ for armchair tubes. From the experimental observations, it can be concluded that in a bundle of armchair SWCNTs, the interaction between the tubes causes a dip in the DOS in individual tube near Fermi level which in turn causes reduction of failure stress and failure strain.

\section{Conclusions}

Hence, this is established in the present work that mechanical properties of the CNTs depend on the type of the CNTs used. A main reason of the mismatch of the experimentally observed values with the theoretical predictions is the types of CNT taken as interlayer interaction plays a vital role in changing the mechanical properties of the CNTs considerably. Composite builders have to keep in mind this effect, while preparing composites with CNTs as the mechanical performance of the composite will vary with the type of CNT used. Consequently, the load transfer efficiency between the CNTs and the polymer matrix will be largely influenced.

Open Access This article is distributed under the terms of the Creative Commons Attribution License which permits any use, distribution, and reproduction in any medium, provided the original author(s) and the source are credited.

\section{References}

Avila AF, Lacerda GSR (2008) Molecular mechanics applied to single-walled carbon nanotubes. Mater Res 11:325-333

Batra RC, Sears A (2007) Uniform radial expansion/contraction of carbon nanotubes and their tranverse elastic moduli. Model Simul Mater Sci Eng 15:835-844

Berendsen HJC, Postma JPM, van Gunsteren WF, DiNola A, Haak JR (1984) Molecular dynamics with coupling to an external bath. J Chem Phys 81:3684-3690

Brenner DW, Shenderova OA, Harrison JA, Stuart SJ, Ni B, Sinnot SB (2002) A second-generation reactive empirical bond order (REBO) potential energy expression for hydrocarbons. J Phys Condens Matter 14:783-802

Coluci VR, Pugno NM, Dantas SO, Galvao DS, Jorio A (2007) Atomistic simulations of the mechanical properties of 'super' carbon nanotubes. Nanotechnology 18:335702 
Connell MJO', Bachilo SM, Huffman CB, Moore VC, Strano MS, Haroz EH, Rialon KL, Boul PJ, Noon WH, Kittrell C, Ma J, Hauge RH, Weisman RB, Smalley RE (2002) Band gap fluorescence from individual single-walled carbon nanotubes. Science 297:593-596

Demczyk BG (2002) Direct mechanical measurement of the tensile strength and elastic modulus of multiwalled carbon nanotubes. Mater Sci Eng, A 334:173-178

Falvo MR, Clary GJ, Taylor RM, Chi V, Brooks FP Jr, Washburn S, Superfine R (1997) Bending and buckling of carbon nanotubes under large strain. Nature 389:582-584

Jorio A, Filho AGS, Dresselhaus G, Dresselhaus MS, Saito R, Hafner JH, Lieber CM, Matinaga FM, Dantas MSS, Pimenta MA (2001) Joint density of electronic states for one isolated single-wall carbon nanotube studied by resonant Raman scattering. Phys Rev B 63:245416

Krishnan A, Dujardin E, Ebbesen TW, Yianilos PN, Treacy MMJ (1998) Young's modulus of single-walled nanotubes. Phys Rev B 58:14013-14019

Lennard-Jones JE (1924) On the determination of molecular fields-II. Proc Royal Soc Lond A 106:463-477

Liew KM, Wong CH, He XQ, Tan MJ, Meguid SA (2004) Nanomechanics of single and multiwalled carbon nanotubes. Phys Rev B 69:115429

Liu JZ, Zheng QS, Wang LF, Jiang Q (2005) Mechanical properties of single-walled carbon nanotube bundles as bulk materials. J Mech Phys Solids 53:123-142

Mashapa MG, Ray SS (2010) Molecular dynamics simulation studies of structural and mechanical properties of single-walled carbon nanotubes. J Nanosci Nanotechnol 10:8083-8090

Mohammadpour E, Awang M, Abdullah MZ (2011) Predicting the Young's Modulus of single-walled carbon nanotubes using finite element modeling. J Appl Sci 11:1653-1657

Nichols JA, Saito H, Deck C, Bandaru PR (2007) Artificial introduction of defects into vertically aligned multiwalled carbon nanotube ensembles: application to electrochemical sensor. J Appl Phys 102:064306

Ouyang M, Huang JL, Cheung CL, Lieber CM (2001) Energy gaps in metallic single-walled carbon nanotube. Science 292:702-705
Ouyang M, Huang JL, Cheung CL, Lieber CM (2002) Fundamental electronic properties and applications of single-walled carbon nanotubes. Acc Chem Res 35:1018-1025

Poncharal P, Wang ZL, Ugarte D, de Heer WA (1999) Electrostatic deflections and electromechanical resonances of carbon nanotubes. Science 283:1513-1516

Reich S, Thomsen C, Ordejon P (2002) Electronic band structure of isolated and bundled carbon nanotubes. Phys Rev B 65:155411

Salvetat JP, Kulik AJ, Bonard JM, Briggs GAD, Stöckli T, Metenier K, Bonnamy S, Beguin F, Burnham NA, Forro L (1999a) Elastic modulus of ordered and disordered multiwalled carbon nanotubes. Adv Mater 11:161-165

Salvetat JP, Brigs GAD, Bonard JM, Bacsa RR, Kulik AJ, Stockli T, Burnhum NA, Forro L (1999b) Elastic and shear moduli of single-walled carbon nanotube ropes. Phys Rev Lett 82:944-947

Tang C, Guo W, Chen C (2011) Structural and mechanical properties of partially unzipped carbon nanotubes. Phys Rev B 83:075410

Treacy MMJ, Ebbesen TW, Gibson JM (1996) Exceptional high Young's modulus observed for individual carbon nanotubes. Nature 381:678-680

Wildoer JWG, Venema LC, Rinzler AG, Smalley RE, Dekker C (1998) Electronic structure of atomically resolved carbon nanotubes. Nature 391:59-62

Wong EW, Sheehan PE, Lieber CM (1997) Nanobeam mechanics: elasticity, strength and toughness of nanorods and nanotubes. Science 277:1971-1975

Xia ZH, Guduru PR, Curtin WA (2007) Enhancing mechanical properties of multiwall carbon nanotubes via sp3 interwall bridging. Phys Rev Lett 98:245501

Yakobson BI, Brabec CJ, Bernholc J (1996) Nanomechanics of carbon tubes: instabilities beyond linear response. Phys Rev Lett 76:2511-2514

Yu MF, Files BS, Arepalli S, Ruoff RS (2000) Tensile loading of ropes of single wall carbon nanotubes and their mechanical properties. Phys Rev Lett 84:5552-5555

Zou J, Huang X, Arroyo M, Zhang S (2009) Effective coarse-grained simulations of super-thick multi-walled carbon nanotubes under torsion. J Appl Phys 105:033516 